\title{
Superoxide Dismutase Activity in Small Mesenteric Arteries Is Downregulated by Angiotensin II but Not by Hypertension
}

\author{
Kyu-Tae Kang', Jennifer C. Sullivan ${ }^{2,3}$ and Jennifer S. Pollock ${ }^{3,4}$ \\ ${ }^{1}$ College of Pharmacy, Duksung Innovative Drug Center, Duksung Women's University, Seoul, Korea \\ ${ }^{2}$ Department of Physiology, Augusta University, Augusta, GA, USA \\ ${ }^{3}$ Medical College of Georgia, Augusta University, Augusta, GA, USA \\ ${ }^{4}$ Section of Cardio-Renal Physiology and Medicine, Division of Nephrology, Department of Medicine, University of \\ Alabama at Birmingham, Birmingham, AL, USA
}

\begin{abstract}
Many studies reported reduced antioxidant capacity in the vasculature under hypertensive conditions. However, little is known about the effects of antihypertensive treatments on the regulation of vascular antioxidant enzymes. Thus, we hypothesized that antihypertensive treatments prevent the reduction of antioxidant enzyme activity and expression in the small vessels of angiotensin II-induced hypertensive rats (ANG). We observed the small mesenteric arteries and small renal vessels of normotensive rats (NORM), ANG, and ANG treated with a triple antihypertensive therapy of reserpine, hydrochlorothiazide, and hydralazine (ANG + TTx). Systolic blood pressure was increased in ANG, which was attenuated by 2 weeks of triple therapy $(127,191$, and $143 \mathrm{mmHg}$ for NORM, ANG, and ANG + TTx, respectively; $p<0.05$ ). Total superoxide dismutase (SOD) activity in the small mesenteric arteries of ANG was lower than that of NORM. The protein expression of SOD1 was lower in ANG than in NORM, whereas SOD2 and SOD3 expression was not different between the groups. Reduced SOD activity and SOD1 expression in ANG was not restored in ANG + TTx. Both SOD activity and SOD isoform expression in the small renal vessels of ANG were not different from those of NORM. Interestingly, SOD activity in the small renal vessels was reduced by TTx. Between groups, there was no difference in catalase activity or expression in both the small mesenteric arteries and small renal vessels. In conclusion, SOD activity in the small mesenteric arteries decreased by angiotensin II administration, but not by hypertension, which is caused by decreased SOD1 expression.
\end{abstract}

Key words: Superoxide dismutase, Angiotensin II, Triple antihypertensive therapy, Small vessels

\section{INTRODUCTION}

In many diseases, an imbalance between prooxidants and antioxidants leads to oxidative stress. Oxidative stress exerts many deleterious effects on the vasculature, including a decrease in nitric oxide (NO) levels, vascular remodeling, and/or vasoconstriction, thereby leading to vascular dysfunction (1). Oxidative stress has been implicated in

Correspondence to: Kyu-Tae Kang, College of Pharmacy, Duksung Innovative Drug Center, Duksung Women's University, 33, Samyangro 144-gil, Dobong gu, Seoul 01369, Korea E-mail: ktkang@duksung.ac.kr

This is an Open-Access article distributed under the terms of the Creative Commons Attribution Non-Commercial License (http:// creativecommons.org/licenses/by-nc/3.0) which permits unrestricted non-commercial use, distribution, and reproduction in any medium, provided the original work is properly cited. the pathogenesis and complications of many cardiovascular diseases, including hypertension, hypercholesterolemia, atherosclerosis, diabetes, and heart failure (2). Increased reactive oxygen species (ROS) production during hypertension has been shown in human patients $(3,4)$ and experimental animal models, such as spontaneously hypertensive rats (SHR) (5), deoxycorticosterone acetate-salt hypertensive rats (6), Dahl salt-sensitive rats (SS) (7), and angio- 
tensin II-induced hypertensive rats (ANG) (8). Specifically, reduced antioxidant capacity appears to contribute to the overproduction of ROS in human patients with hypertension (9), SHR (10), SS (11), diabetic rats (12), and ANG (13).

Our laboratory has reported that the small arteries of ANG show increased oxidative stress and reduced NO signaling (14). Moreover, we also showed previously that an antihypertensive triple therapy with reserpine, hydrochlorothiazide, and hydralazine restored NO signaling (15). The activity and protein expression of antioxidant enzymes in small vessels have not been investigated as intensively as those in conduit vessels. Furthermore, very little information is available regarding the relationship between antihypertensive treatments and antioxidant enzyme regulation. Thus, we aimed to investigate the antioxidant capacity of small vessels under hypertensive conditions.

Superoxide dismutase (SOD) is an antioxidant enzyme that catalyzes the dismutation of $\mathrm{O}_{2}^{-}$to $\mathrm{H}_{2} \mathrm{O}_{2} . \mathrm{H}_{2} \mathrm{O}_{2}$ is then converted to $\mathrm{O}_{2}$ and $\mathrm{H}_{2} \mathrm{O}$ by catalase. Three isoforms of SOD have been identified: intracellular copper zinc SOD (CuZn-SOD; SOD1), mitochondrial manganese SOD (Mn-SOD; SOD2), and extracellular CuZn-SOD (ecSOD; SOD3). We hypothesized that reduced antioxidant capacity in the small vessels of ANG will be restored by antihypertensive treatments. We used ANG in this study because we also used this rat model in our previous studies on small arteries $(14,15)$. The activities of SOD isoforms and catalase as well as each protein expression were evaluated in the small mesenteric arteries and small renal vessels of normotensive rats (NORM), ANG, and ANG treated with triple antihypertensive therapy (reserpine, hydrochlorothiazide, and hydralazine) (ANG + TTx).

\section{MATERIALS AND METHODS}

Materials. Angiotensin II was purchased from Phoenix Pharmaceuticals, Inc (Belmont, CA, USA). Mini-osmotic pumps were purchased from Alzet (Model 2002, Cupertino, CA, USA). All other chemicals were purchased from Sigma-Aldrich (St. Louis, MO, USA).

Animal model. Male Sprague-Dawley rats weighing 200-250 g (Charles River Laboratories, Wilmington, MA, USA) were divided into 3 groups: NORM, ANG and ANG + TTx $(30 \mathrm{mg} / \mathrm{kg} /$ day hydralazine, $10 \mathrm{mg} / \mathrm{kg} /$ day hydrochlorothiazide, $0.5 \mathrm{mg} / \mathrm{kg} /$ day reserpine). The dose and route of administration of the triple therapy were previously shown to be efficacious (16-19). To establish angiotensin II-induced hypertension, an osmotic mini-pump containing angiotensin II was implanted subcutaneously into rats under isoflurane anesthesia (IsoFlo; Abbott Laboratories, North Chicago, IL, USA). Angiotensin II was induced chronically at the rate of $70 \mathrm{ng} / \mathrm{min}$ for 2 weeks. Triple therapy (TTx) was incorporated in the drinking water 3 days prior to and throughout the 2-week period of angiotensin II infusion. The TTx solution was changed every three days and modified each drug concentration according to the water intake of each rat. Systolic blood pressure was measured after 7 and 14 days of angiotensin II infusion by tail-cuff plethysmography, as previously described (20). Systolic blood pressure of each rat was determined in the average of 4 to 6 independent readings. After 2 weeks, the rats were anesthetized with sodium pentobarbital (Nembutal, $50 \mathrm{mg} / \mathrm{kg}$ IP; Abbott Laboratories), and the small mesenteric arteries and small renal vessels were isolated, as previously described $(21,22)$. Briefly, the mesenteric bed, including arteries and veins, was cut away from the intestinal wall, and placed in a dissecting dish containing ice-cold physiological saline solution (PSS; $\mathrm{pH}=$ $7.4 ; 130 \mathrm{mmol} / \mathrm{L} \mathrm{NaCl}, 4.7 \mathrm{mmol} / \mathrm{L} \mathrm{KCl}, 1.18 \mathrm{mmol} / \mathrm{L}$ $\mathrm{KH}_{2} \mathrm{PO}_{4}, 1.17 \mathrm{mmol} / \mathrm{L} \mathrm{MgSO}{ }_{4} \cdot 7 \mathrm{H}_{2} \mathrm{O}, 14.9 \mathrm{mmol} / \mathrm{L} \mathrm{NaHCO}_{3}$, $5.5 \mathrm{mmol} / \mathrm{L}$ dextrose, $0.26 \mathrm{mmol} / \mathrm{L}$ EDTA, $1.6 \mathrm{mmol} / \mathrm{L}$ $\mathrm{CaCl}_{2}$ ). The fat was carefully pulled off from the mesenteric beds, and then the veins were removed using an Olympus dissecting microscope (Center Valley, PA, USA). For small renal vessels, kidneys were removed and immediately placed in a dissecting dish containing ice-cold physiological saline solution. After removal of the renal capsule, the kidney was placed between a circle sieve of 70-mm pore size (Biodesign, Carmel, NY, USA). Small renal vessels were immediately isolated by rapid and gentle grating under the microscope. Isolated small mesenteric arteries and small renal vessels were subsequently frozen in liquid nitrogen and stored at $-80^{\circ} \mathrm{C}$ until further use. All experiments were conducted in accordance with the Guide for the Care and Use of Laboratory Animals of the National Institutes of Health and were approved and monitored by the Augusta University Institutional Animal Care and Use Committee.

Measurement of antioxidant enzyme activity. Small mesenteric arteries and small renal vessels were isolated in cold physiological saline solution (PSS; same composition as that in the previous section), as previously described $(15,21,22)$. Isolated vascular beds were placed into icecold homogenization buffer $(20.0 \mathrm{mmol} / \mathrm{L} \mathrm{Na-HEPES}$, $\mathrm{pH}=7.4 ; 1.0 \mathrm{mmol} / \mathrm{L}$ EGTA; $210.0 \mathrm{mmol} / \mathrm{L}$ mannitol; $70.0 \mathrm{mmol} / \mathrm{L}$ sucrose) and homogenized on ice with a glass tissue grinder. Supernatants were obtained by centrifugation $(1,500 \times g, 5 \mathrm{~min}$ for SOD; $10,000 \times g, 15 \mathrm{~min}$ for catalase), and protein concentration was determined by standard Bradford assay. SOD and catalase activities were determined according to the manufacturers' instructions (Cayman Chemical, Ann Arbor, MI, USA), and normalized to the total protein concentration of each sample.

Immunoblotting. The small mesenteric arteries and small renal vessels were placed into ice-cold homogenization buffer (same composition as that in the previous sec- 
tion) containing protease inhibitors (Roche Applied Science, Indianapolis, IN, USA) and phosphatase inhibitors (Pierce, Pockford, IL, USA), and homogenized on ice with a glass tissue grinder. Protein concentration was determined by standard Bradford assay and western blotting, as described previously (23). Briefly, after transferring the proteins onto a PVDF membrane, the membrane was blocked in a blocking buffer (LI-COR Biosciences, Lincoln, NE, USA). Two-color immunoblotting was performed using primary antibodies against SOD1 (Stressgen, Victoria, British Columbia Canada), SOD2 (Stressgen, Victoria, British Columbia Canada), SOD3 (generously provided by Dr. Tohru Fukai, Augusta University), catalase (Abcam, Cambridge, MA, USA), and $\beta$-actin (Sigma-Aldrich). Specific bands were detected using an Odyssey Infrared Imager (LI-COR Biosciences, Lincoln, NE, USA). IRDye800

(A)

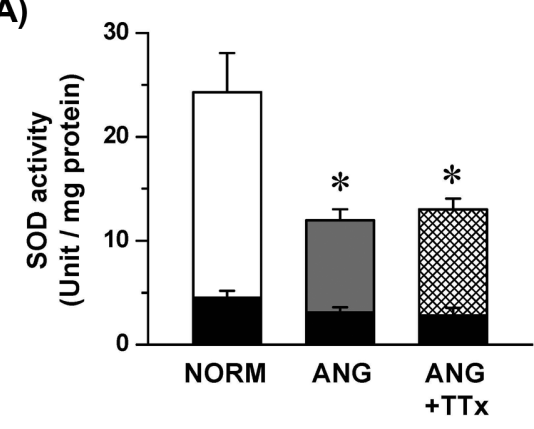

(C)

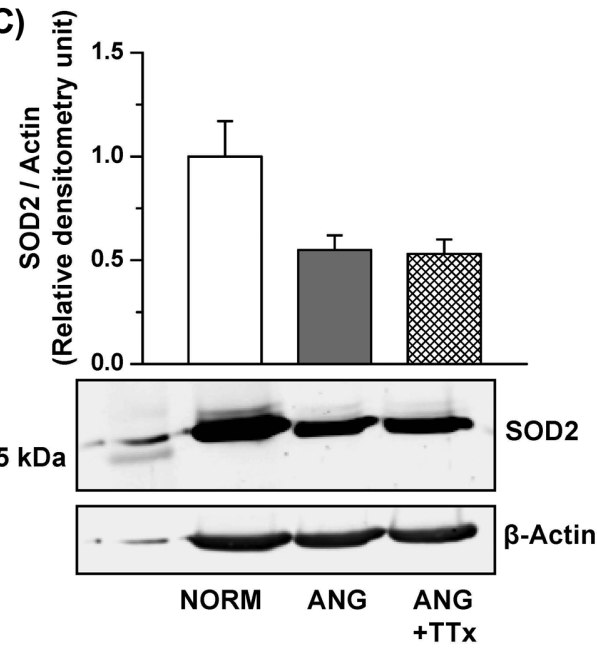

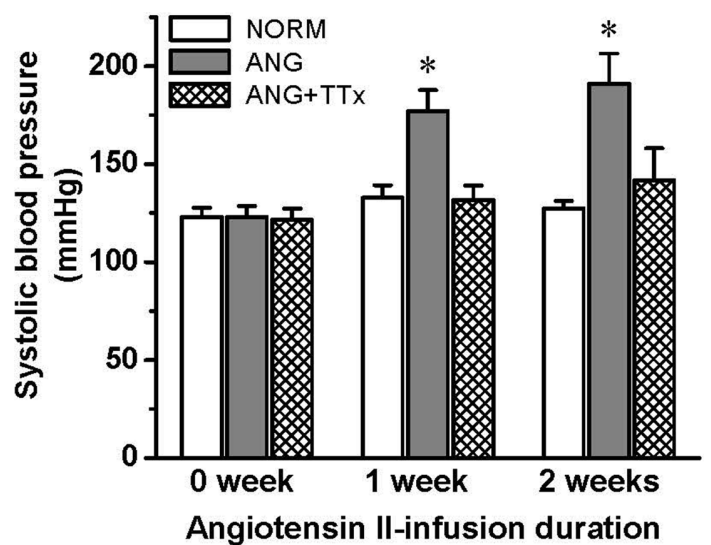

Fig. 1. Systolic blood pressure of NORM, ANG, and ANG + TTx ( $n=7$ per group) before and after 1 and 2 weeks of angiotensin II infusion. ${ }^{*} p \leq 0.05$ vs. NORM at each period.

(B)

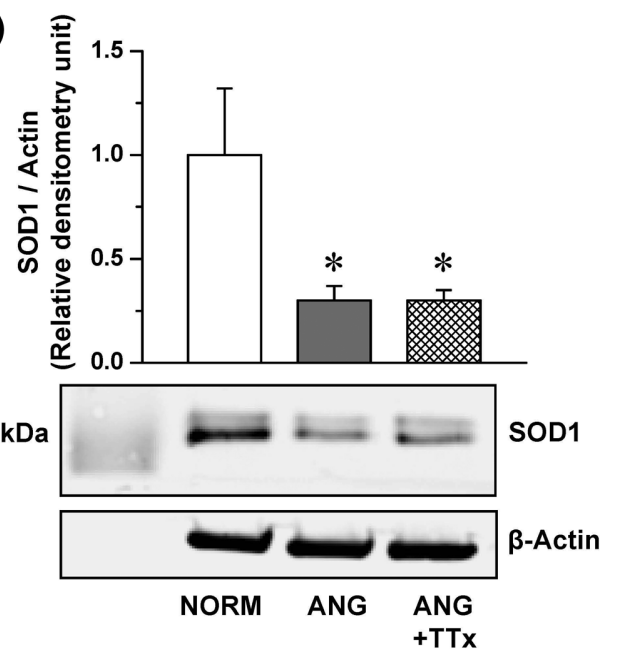

(D)

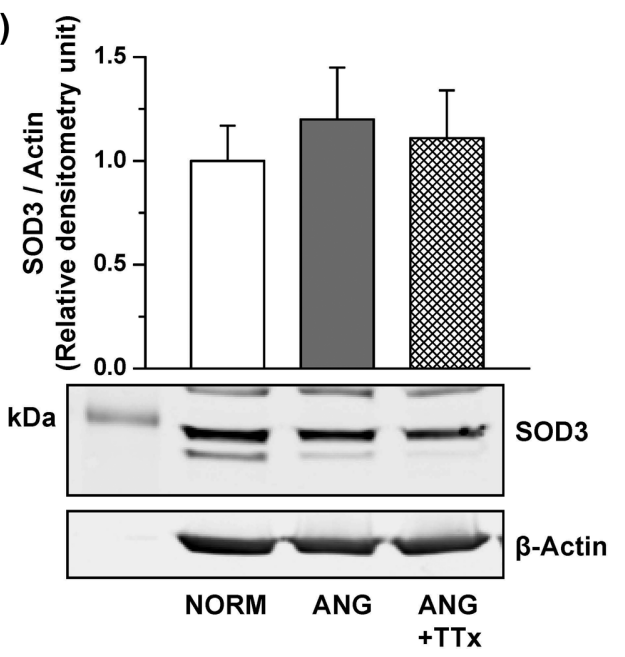

Fig. 2. Superoxide dismutase (SOD) activity $(A ; n=5-6)$ and protein expression of SOD1 $(B ; n=4-5)$, SOD2 $(C ; n=5-7)$, and SOD3 (D; $n=4-5$ ) in the small mesenteric arteries of NORM, ANG, and ANG + TTx. (A) SOD activity was normalized to mg protein, and black inserted bars are SOD activity with potassium cyanide treatment. $(B, C, D)$ Relative densitometric units of the enzymes were normalized to that of $\beta$-actin. " $p \leq 0.05$ vs. NORM. 
(Rockland Immunochemicals, Limerick, PA, USA) and AlexaFluor 680 (Molecular Probes, Eugene, OR, USA) were used as secondary antibodies to detect two primary antibodies from different species. Relative densitometric units of the proteins were calculated via normalization to that of $\beta$-actin.

Statistical analysis. Data are expressed as mean \pm SEM and were analyzed by ANOVA followed by Bonferroni's correction for multiple comparisons using the Prism (GraphPad Software, La Jolla, CA, USA). $p$ value of $\leq 0.05$ was considered statistically significant.

\section{RESULTS}

Systolic blood pressure. Systolic blood pressure was significantly higher in ANG than in NORM after one

(A)

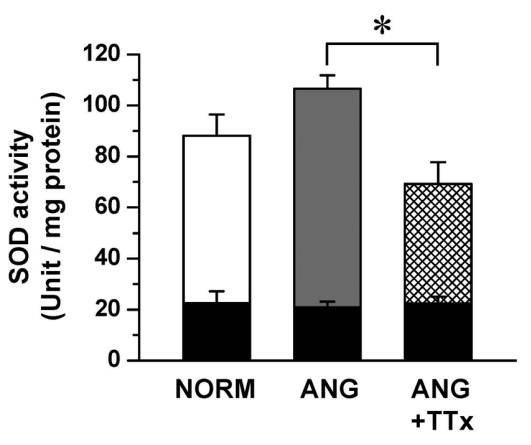

(C)

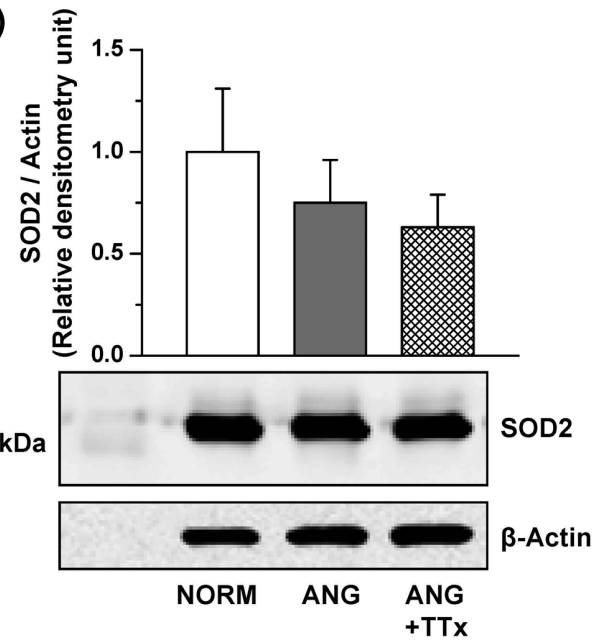

week of angiotensin II infusion, and this increased pressure was maintained at the second week of angiotensin II infusion ( $\mathrm{n}=7$; Fig. 1). However, TTx blocked the increase in systolic blood pressure for 2 weeks of treatment.

SOD activity and expression in the small mesenteric arteries. Total SOD activity in the small mesenteric arteries of ANG was lower than that of NORM (Fig. 2A), and this reduction was also observed in ANG + TTx (Fig. 2A). Potassium cyanide inhibits SOD1 and SOD3 activity (24); thus, the vessels were incubated with potassium cyanide to measure SOD2 activity. SOD2 activity was similar between groups (black inserted bars in Fig. 2A), indicating that the activity of SOD1 and SOD3, not SOD2, in the small mesenteric arteries were affected by angiotensin II-induced hypertension. SOD1 expression was lower in ANG and ANG + TTx than in NORM (Fig. 2B), and
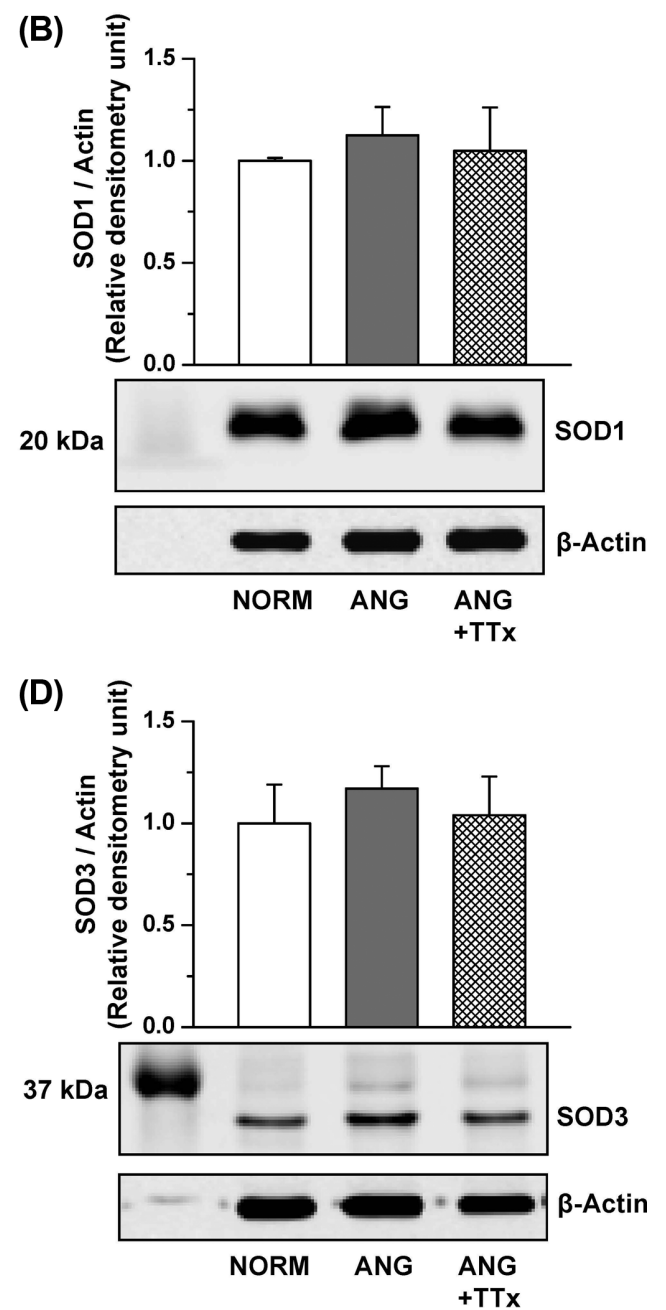

Fig. 3. Superoxide dismutase (SOD) activity $(A ; n=4-5)$ and protein expression of SOD1 ( $B ; n=5-6)$, SOD2 (C; $n=6)$, and SOD3 (D; $\mathrm{n}=6$ ) in the small renal vessels from NORM, ANG, and ANG + TTx. (A) SOD activity was normalized to mg protein, and black inserted bars are SOD activity with potassium cyanide treatment. $(B, C, D)$ Relative densitometric unit units of the enzymes were normalized to that of $\beta$-actin. ${ }^{*} p \leq 0.05$ vs. ANG. 
this downregulation of SOD expression was correlated with reduced SOD activity. The expression of SOD2 and SOD3 was not statistically different between groups (Fig. 2C, 2D, respectively).

SOD activity and expression in small renal vessels. Total SOD activity in the small renal vessels of ANG was not different from that of NORM (Fig. 3A). Interestingly, a reduction of SOD activity was observed in ANG + TTx (Fig. 3A). SOD2 activity after potassium cyanide incubation was similar between groups (black inserted bars in Fig. 3A). The expression of all three SOD isoforms were comparable between groups (Fig. 3B-3D).

\section{Catalase activity and expression in the small mesen-} teric arteries and small renal vessels. Both the enzymatic activity and protein expression of catalase in the small mesenteric arteries were comparable between groups (Fig. 4A, 4B). Similarly, there was no change in catalase activity or protein expression in the small renal vessels between groups (Fig. 5A, 5B).
(A)

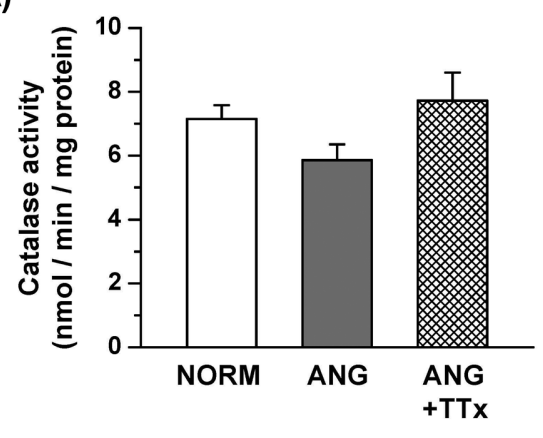

(B)

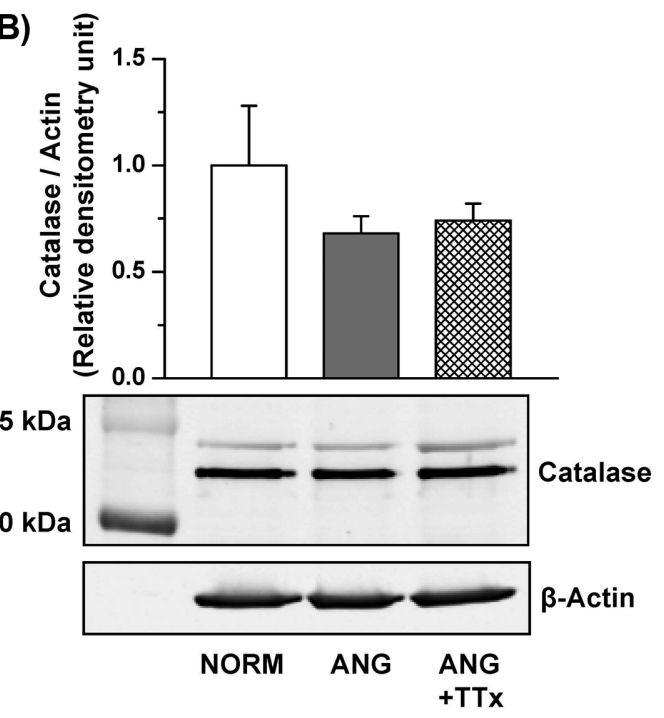

Fig. 4. Catalase activity $(A ; n=5-6)$ and protein expression $(B ; n=3-5)$ in the small mesenteric arteries of NORM, ANG, and ANG + TTx. (A) Catalase activity was normalized to mg protein. (B) Relative densitometric unit of catalase were normalized to that of $\beta$-actin.

(A)

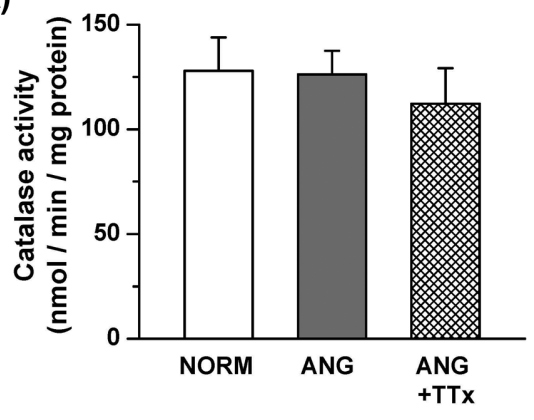

(B)

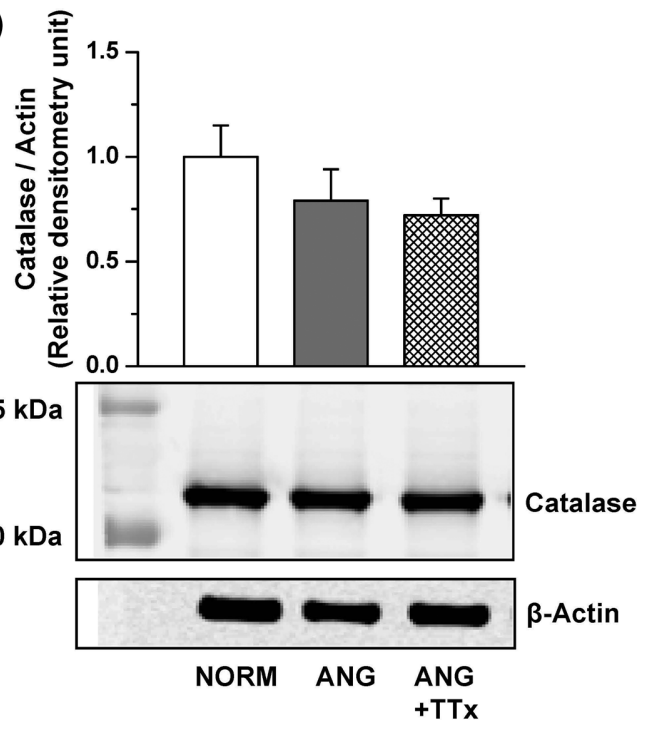

Fig. 5. Catalase activity $(A ; n=7)$ and protein expression $(B ; n=6)$ in the small renal vessels of NORM, $A N G$, and $A N G+T T x$. (A) Catalase activity was normalized to mg protein. (B) Relative densitometric unit of catalase were normalized to that of $\beta$-actin. 


\section{DISCUSSION}

We hypothesized that angiotensin II-induced hypertension reduces antioxidant capacity, and antihypertensive treatment with ANG will restore antioxidant capacity in small vessels. We found that TTx significantly blunted the rise in blood pressure. However, reduced SOD activity and SOD1 expression in the small mesenteric arteries of ANG were not improved by this treatment. There was no difference in total SOD activity and expression of three SOD isoforms in the small renal vessels between ANG and NORM. Unexpectedly, we observed a decrease in SOD activity in the small renal vessels of ANG + TTx. The activity and expression of catalase in either vascular bed were not different between groups. These data indicated that SOD1 expression, and most likely SOD activity as well, in the small mesenteric arteries is downregulated by angiotensin II, but not high blood pressure. In contrast, antioxidant enzymes in the small renal vessels appeared to be resistant to angiotensin II and hypertension.

Angiotensin II is a well-recognized activator of the nicotinamide adenine dinucleotide phosphate (NADPH) oxidase, which is a major source of $\mathrm{O}_{2}^{-}$in the vascular system $(25,26)$. Enzymes such as NADPH oxidase and uncoupled nitric oxide synthase $3(\mathrm{NOS} 3)$ produce $\mathrm{O}_{2}^{-}$or $\mathrm{H}_{2} \mathrm{O}_{2}$ mainly within the cell (27); thus, SOD activity in the intracellular space would be critical for lowering oxidative stress during angiotensin II activation. SOD1 is localized in the cellular cytosolic compartment. Transgenic mice overexpressing SOD1 showed an improvement in angiotensin II-mediated endothelial function and a decrease in angiotensin II-induced $\mathrm{O}_{2}^{-}$production (28). Moreover, the infusion of recombinant SOD1 reduced blood pressure in ANG (29). Consistent with the roles of SOD1 reported in other studies, reduced SOD activity in the small mesenteric arteries of ANG was correlated with reduced SOD1 expression. We previously reported that $\mathrm{H}_{2} \mathrm{O}_{2}$ is increased in the small mesenteric arteries of ANG (14). $\mathrm{H}_{2} \mathrm{O}_{2}$ has been suggested to inactivate $\mathrm{CuZn}$ SODs (SOD1 and SOD3) by reacting with the $\mathrm{Cu}$ center of the enzyme, leading to the formation of $\mathrm{Cu}-\mathrm{OH}$ radical (30). Therefore, increased $\mathrm{H}_{2} \mathrm{O}_{2}$ production in the small mesenteric arteries of ANG may reduce the activity of SOD1 by negative feedback mechanism.

Our previous study also showed reduced NO/cGMP signaling in the small mesenteric arteries of ANG and that antihypertensive treatment restored NO/cGMP signaling, which correlated with an increased biosynthesis of tetrahydrobiopterin $\left(\mathrm{BH}_{4}\right)$, an essential cofactor for NO production from NOS (15). Interestingly, the increased level of oxidized $\mathrm{BH}_{4}, 7,8$-dihydrobiopterin $\left(\mathrm{BH}_{2}\right)$, is not affected by antihypertensive treatment in ANG (15). These results correlate with our present finding of continued suppression of SOD activity with antihypertensive treatment. Taken together, we suggest that NO/cGMP signaling in the small mesenteric arteries can be restored by antihypertensive treatment (15) by increasing $\mathrm{BH}_{4}$ biosynthesis, not restoring antioxidant capacity.

Our findings highlighted a difference in the regulation of SOD activity and SOD1 expression between the small mesenteric arteries and small renal vessels under the same angiotensin II-induced hypertensive conditions. Although this study appears to be the only one investigating the regulation of antioxidant enzymes in small renal vessels, others have shown distinct regulation of antioxidant enzymes between conduit and mesenteric arteries (31) or renal cortex and medulla $(32,33)$. Specifically, Gongora et al. showed that chronic angiotensin II infusion in mice increased SOD3 activity and expression in the aorta, whereas SOD1 and SOD2 activities were unchanged (31); moreover, in the mesenteric arteries, angiotensin II infusion increased SOD3 and SOD1 expression, but did not alter SOD2 expression (31). Compared with those observed in our study, SOD activity and expression are distinctly regulated within each vascular bed and between species. In terms of species, mouse SOD3 is a tetramer, whereas rat SOD3 is a dimer, and this structural difference causes a lack of affinity for heparin sulfate proteoglycans in rats (34). Thus, the protein level of SOD3 in rat vasculature is relatively lower than that in most other species (35), and the role of vascular SOD3 in rats may be not as important as in other species (36). Indeed, exogenous SOD3 treatment shows a beneficial effect in hypertensive rats, but not in hypertensive mice (37).

We observed no change in either the activity or protein expression of SOD2 in the small mesenteric arteries or small renal vessels between all groups. Other studies also showed no change in SOD2 in the aorta of $2 \mathrm{~K} 1 \mathrm{C}$ rats (37), and in the aorta and mesenteric arteries of angiotensin IIinduced hypertensive mice $(31,38)$, renal cortex, and renal medulla of angiotensin II-induced hypertensive mice $(13,33)$. Accordingly, SOD2 may play a pivotal role in cell survival because SOD2 protects mitochondrial function by eliminating mitochondria-derived $\mathrm{O}_{2}^{-}$, but not in response to an angiotensin II-mediated increase in oxidative stress in the vasculature.

Total SOD activity in the small renal vessels of ANG was not different from that of NORM. Intriguingly, SOD activity in the small renal vessels of ANG + TTx was lower than that of ANG. It is not clear why SOD activity in the small renal vessels was reduced by TTx, but one speculation is that the antioxidant effects of hydralazine (39) may contribute to the antioxidant system of renal vessels, thereby lowering the activity of residential SODs. Further investigation is necessary to reveal the specific mechanism. The protein expression of the three SODs in the small renal vessels was not different between groups, indicating that SODs in the renal vasculature were resis- 
tant to angiotensin II induction and high blood pressure.

Catalase is a peroxidase that eliminate $\mathrm{H}_{2} \mathrm{O}_{2}$ in the cells by converting it to water and oxygen. Reduced catalase activity can lead to the accumulation of $\mathrm{H}_{2} \mathrm{O}_{2}$ in the tissues. Our present data showed that both the activity and protein expression of catalase in both the small mesenteric arteries and small renal vessels were not different between experimental groups. Interestingly, we previously showed increased $\mathrm{H}_{2} \mathrm{O}_{2}$ production in the small mesenteric arteries of ANG, but not of NORM (14). Thus, we speculate that increased $\mathrm{H}_{2} \mathrm{O}_{2}$ in the small mesenteric arteries of ANG may be due to both the observed prooxidant enzymatic sources of $\mathrm{H}_{2} \mathrm{O}_{2}$ production, such as NOS-derived $\mathrm{H}_{2} \mathrm{O}_{2}$, and reduced SOD activity, regardless of intact catalase activity and expression.

Vascular ROS level is regulated by the balance between the production and elimination of ROS. Increased $\mathrm{O}_{2}^{-}$production has gained attention in the study of endothelial dysfunction because of its ability to react with NO, resulting in $\mathrm{ONOO}^{-}$production. $\mathrm{ONOO}^{-}$is a potent oxidizing agent causing deleterious lipid peroxidation and membrane damage in vascular cells. Therefore, SODs may be critical to protect $\mathrm{NO}$ bioavailability and prevent $\mathrm{ONOO}^{-}$ generation in the blood vessels. Our data showed that SOD1 activity and expression in the small mesenteric arteries was downregulated, which was likely attributed to angiotensin II-dependent vascular dysfunction. In contrast, SOD activity and expression in the small renal vessels remained unchanged. This differential regulation of SOD highlighted a distinct difference in the importance of antioxidant system in each vascular bed.

\section{ACKNOWLEDGMENTS}

This research was supported by the National Institutes of Health (NIH P01 HL69999 and NIH P01 HL95499), and also supported by Basic Science Research Program through the National Research Foundation of Korea (NRF) funded by the Ministry of Education (2016R1A6A1A03007648) and the Ministry of Science, ICT \& Future Planning (2013R1A1A1062132 and 2017R1A2B4005463).

\section{CONFLICT OF INTEREST}

The authors declare no conflict of interest.

Received August 31, 2018; Revised September 9, 2018; Accepted September 10, 2018

\section{REFERENCES}

1. Lee, M.Y. and Griendling, K.K. (2008) Redox signaling, vascular function, and hypertension. Antioxid. Redox. Signal., 10, 1045-1059.
2. Cai, H. and Harrison, D.G. (2000) Endothelial dysfunction in cardiovascular diseases: the role of oxidant stress. Circ. Res., 87, 840-844.

3. Lip, G.Y., Edmunds, E., Nuttall, S.L., Landray, M.J., Blann, A.D. and Beevers, D.G. (2002) Oxidative stress in malignant and non-malignant phase hypertension. J. Hum. Hypertens., 16, 333-336.

4. Higashi, Y., Sasaki, S., Nakagawa, K., Matsuura, H., Oshima, T. and Chayama, K. (2002) Endothelial function and oxidative stress in renovascular hypertension. N. Engl. J. Med., 346, 1954-1962.

5. Wu, R., Millette, E., Wu, L. and de Champlain, J. (2001) Enhanced superoxide anion formation in vascular tissues from spontaneously hypertensive and desoxycorticosterone acetate-salt hypertensive rats. J. Hypertens., 19, 741-748.

6. Beswick, R.A., Zhang, H., Marable, D., Catravas, J.D., Hill, W.D. and Webb, R.C. (2001) Long-term antioxidant administration attenuates mineralocorticoid hypertension and renal inflammatory response. Hypertension, 37, 781-786.

7. Uehara, Y., Numabe, A., Hirawa, N., Kawabata, Y., Iwai, J., Ono, H., Matsuoka, H., Takabatake, Y., Yagi, S. and Sugimoto, T. (1991) Antihypertensive effects of cicletanine and renal protection in Dahl salt-sensitive rats. J. Hypertens., 9, 719-728.

8. Rajagopalan, S., Kurz, S., Munzel, T., Tarpey, M., Freeman, B.A., Griendling, K.K. and Harrison, D.G. (1996) Angiotensin II-mediated hypertension in the rat increases vascular superoxide production via membrane NADH/NADPH oxidase activation. Contribution to alterations of vasomotor tone. J. Clin. Invest., 97, 1916-1923.

9. Russo, C., Olivieri, O., Girelli, D., Faccini, G., Zenari, M.L., Lombardi, S. and Corrocher, R. (1998) Anti-oxidant status and lipid peroxidation in patients with essential hypertension. J. Hypertens., 16, 1267-1271.

10. Ulker, S., McMaster, D., McKeown, P.P. and Bayraktutan, U. (2003) Impaired activities of antioxidant enzymes elicit endothelial dysfunction in spontaneous hypertensive rats despite enhanced vascular nitric oxide generation. Cardiovasc. Res., 59, 488-500.

11. Meng, S., Roberts, L.J., 2nd, Cason, G.W., Curry, T.S. and Manning, R.D., Jr. (2002) Superoxide dismutase and oxidative stress in Dahl salt-sensitive and -resistant rats. Am. J. Physiol. Regul. Integr. Comp. Physiol., 283, R732- R738.

12. Lewis, P., Stefanovic, N., Pete, J., Calkin, A.C., Giunti, S., Thallas-Bonke, V., Jandeleit-Dahm, K.A., Allen, T.J., Kola, I., Cooper, M.E. and de Haan, J.B. (2007) Lack of the antioxidant enzyme glutathione peroxidase-1 accelerates atherosclerosis in diabetic apolipoprotein E-deficient mice. Circulation, 115, 2178-2187.

13. Vera, T., Kelsen, S., Yanes, L.L., Reckelhoff, J.F. and Stec, D.E. (2007) HO-1 induction lowers blood pressure and superoxide production in the renal medulla of angiotensin II hypertensive mice. Am. J. Physiol. Regul. Integr. Comp. Physiol., 292, R1472-R1478.

14. Kang, K.T., Sullivan, J.C., Sasser, J.M., Imig, J.D. and Pollock, J.S. (2007) Novel nitric oxide synthase--dependent mechanism of vasorelaxation in small arteries from hypertensive rats. Hypertension, 49, 893-901.

15. Kang, K.T., Sullivan, J.C., Spradley, F.T., d’Uscio, L.V., 
Katusic, Z.S. and Pollock, J.S. Antihypertensive therapy increases tetrahydrobiopterin levels and NO/cGMP signaling in small arteries of angiotensin II-infused hypertensive rats. Am. J. Physiol. Heart. Circ. Physiol., 300, H718-H724.

16. Inscho, E.W., Cook, A.K., Murzynowski, J.B. and Imig, J.D. (2004) Elevated arterial pressure impairs autoregulation independently of AT(1) receptor activation. J. Hypertens., 22, 811-818.

17. Vanourkova, Z., Kramer, H.J., Huskova, Z., Vaneckova, I., Opocensky, M., Chabova, V.C., Tesar, V., Skaroupkova, P., Thumova, M., Dohnalova, M., Mullins, J.J. and Cervenka, L. (2006) AT1 receptor blockade is superior to conventional triple therapy in protecting against end-organ damage in Cypla1-Ren-2 transgenic rats with inducible hypertension. J. Hypertens., 24, 2465-2472.

18. Kase, H., Hashikabe, Y., Uchida, K., Nakanishi, N. and Hattori, Y. (2005) Supplementation with tetrahydrobiopterin prevents the cardiovascular effects of angiotensin II-induced oxidative and nitrosative stress. J. Hypertens., 23, 13751382.

19. Landmesser, U., Dikalov, S., Price, S.R., McCann, L., Fukai, T., Holland, S.M., Mitch, W.E. and Harrison, D.G. (2003) Oxidation of tetrahydrobiopterin leads to uncoupling of endothelial cell nitric oxide synthase in hypertension. $J$. Clin. Invest., 111, 1201-1209.

20. Pollock, D.M. and Rekito, A. (1998) Hypertensive response to chronic NO synthase inhibition is different in SpragueDawley rats from two suppliers. Am. J. Physiol., 275, R1719-R1723.

21. Sullivan, J.C., Pollock, D.M. and Pollock, J.S. (2002) Altered nitric oxide synthase 3 distribution in mesenteric arteries of hypertensive rats. Hypertension, 39, 597-602.

22. Schneider, M.P., Wach, P.F., Durley, M.K., Pollock, J.S. and Pollock, D.M. (2010) Sex differences in acute ANG II-mediated hemodynamic responses in mice. Am. J. Physiol. Regul. Integr. Comp. Physiol., 299, R899-R906.

23. Sasser, J.M., Sullivan, J.C., Hobbs, J.L., Yamamoto, T., Pollock, D.M., Carmines, P.K. and Pollock, J.S. (2007) Endothelin A receptor blockade reduces diabetic renal injury via an anti-inflammatory mechanism. J. Am. Soc. Nephrol., 18, 143-154.

24. Westman, G. and Marklund, S.L. (1980) Diethyldithiocarbamate, a superoxide dismutase inhibitor, decreases the radioresistance of Chinese hamster cells. Radiat. Res., 83, 303311.

25. Griendling, K.K., Sorescu, D. and Ushio-Fukai, M. (2000) $\mathrm{NAD}(\mathrm{P}) \mathrm{H}$ oxidase: role in cardiovascular biology and disease. Circ. Res., 86, 494-501.

26. Kim, M., Han, C.H. and Lee, M.Y. (2014) NADPH oxidase and the cardiovascular toxicity associated with smoking. Toxicol. Res., 30, 149-157.
27. Griendling, K.K., Minieri, C.A., Ollerenshaw, J.D. and Alexander, R.W. (1994) Angiotensin II stimulates NADH and NADPH oxidase activity in cultured vascular smooth muscle cells. Circ. Res., 74, 1141-1148.

28. Didion, S.P., Kinzenbaw, D.A. and Faraci, F.M. (2005) Critical role for $\mathrm{CuZn}$-superoxide dismutase in preventing angiotensin II-induced endothelial dysfunction. Hypertension, 46, 1147-1153.

29. Fukui, T., Ishizaka, N., Rajagopalan, S., Laursen, J.B., Capers, Q., Taylor, W.R., Harrison, D.G., de Leon, H., Wilcox, J.N. and Griendling, K.K. (1997) p22phox mRNA expression and NADPH oxidase activity are increased in aortas from hypertensive rats. Circ. Res., 80, 45-51.

30. Kim, S.M. and Kang, J.H. (1997) Peroxidative activity of human Cu,Zn-superoxide dismutase. Mol. Cells, 7, 120-124.

31. Gongora, M.C., Qin, Z., Laude, K., Kim, H.W., McCann, L., Folz, J.R., Dikalov, S., Fukai, T. and Harrison, D.G. (2006) Role of extracellular superoxide dismutase in hypertension. Hypertension, 48, 473-481.

32. Zhan, C.D., Sindhu, R.K., Pang, J., Ehdaie, A. and Vaziri, N.D. (2004) Superoxide dismutase, catalase and glutathione peroxidase in the spontaneously hypertensive rat kidney: effect of antioxidant-rich diet. J. Hypertens., 22, 2025-2033.

33. Welch, W.J., Chabrashvili, T., Solis, G., Chen, Y., Gill, P.S., Aslam, S., Wang, X., Ji, H., Sandberg, K., Jose, P. and Wilcox, C.S. (2006) Role of extracellular superoxide dismutase in the mouse angiotensin slow pressor response. Hypertension, 48, 934-941.

34. Carlsson, L.M., Marklund, S.L. and Edlund, T. (1996) The rat extracellular superoxide dismutase dimer is converted to a tetramer by the exchange of a single amino acid. Proc. Natl. Acad. Sci. U.S.A., 93, 5219-5222.

35. Karlsson, K. and Marklund, S.L. (1988) Extracellular superoxide dismutase in the vascular system of mammals. Biochem. J., 255, 223-228.

36. Marklund, S.L. (1984) Extracellular superoxide dismutase and other superoxide dismutase isoenzymes in tissues from nine mammalian species. Biochem. J., 222, 649-655.

37. Jung, O., Marklund, S.L., Geiger, H., Pedrazzini, T., Busse, R. and Brandes, R.P. (2003) Extracellular superoxide dismutase is a major determinant of nitric oxide bioavailability: in vivo and ex vivo evidence from ecSOD-deficient mice. Circ. Res., 93, 622-629.

38. Fukai, T., Siegfried, M.R., Ushio-Fukai, M., Griendling, K.K. and Harrison, D.G. (1999) Modulation of extracellular superoxide dismutase expression by angiotensin II and hypertension. Circ. Res., 85, 23-28.

39. Daiber, A., Mulsch, A., Hink, U., Mollnau, H., Warnholtz, A., Oelze, M. and Munzel, T. (2005) The oxidative stress concept of nitrate tolerance and the antioxidant properties of hydralazine. Am. J. Cardiol., 96, 25i-36i. 